\title{
Intervención de la terapia del lenguaje en las alteraciones deglutorias y de la voz en pacientes con cáncer de cabeza y cuello.
}

Intervention of language therapy in the alterations of deglutition and voice in patients with head and neck cancer.

Filiación:

Terapeuta de Lenguaje independiente, San José, Costa Rica.

Escuela Terapia de Lenguaje, Universidad Santa Paula, San José, Costa Rica.

Correspondencia: $\square$ Ingrid Mora Umaña. Correo electrónico: ingrid1886@gmail.com

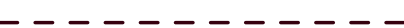

Financiamiento:

Ninguno

Conflictos de Interés:

Ninguno

Forma de citar: Alvarado Murillo

A, López Torres C, Mora Umaña

I, Solano Rojas C. Intervención de la Terapia del Lenguaje en las alteraciones deglutorias y de la voz en pacientes con cáncer de cabeza y cuello. Rev Ter. 2020;14(2):56-70

Abreviaturas: $A D$ : Antes de la deglución; ASCO: Sociedad Americana de Oncología Clínica, por siglas en inglés; CCC: Cáncer de cabeza y cuello; CV: Cuerdas vocales; DD: Durante la deglución; DED: Después de la deglución; INEC: Instituto Nacional de Estadísticas y Censos; OMS: Organización Mundial de la Salud; SEOM: Sociedad Española de Oncología Médica; VA: Vía aérea; VEB: Virus de Epstein-Barr; VPH: Virus del Papiloma Humano

Fecha de recepción: 3 de diciembre del 2019.

Fecha de aceptación: 12 de mayo del 2020.

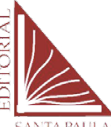

Alexandra Alvarado Murillo ${ }^{1}$, Carolina López Torres ${ }^{1}$, Ingrid Mora Umaña ${ }^{1} \square$, Carolina Solano Rojas ${ }^{2}$

\section{Resumen}

Introducción: En Costa Rica, la incidencia de cáncer de cabeza y cuello para el año 2016 fue de 68,3\%, con una mortalidad por esta causa del 25,4\%. Este tipo de cáncer se origina en las vías aerodigestivas, tiroides y glándulas salivares y genera alteraciones muy importantes en la deglución y la generación de la voz. Este artículo tiene como objetivo definir la intervención de terapia del lenguaje en las alteraciones deglutorias y de la voz en pacientes con cáncer de cabeza y cuello. Metodología: Se realizó una revisión narrativa basada en la revisión bibliográfica de artículos de revisión en inglés y español, libros publicados a partir del año 2000 al 2019 y el criterio de expertos, por ejemplo, terapeutas del lenguaje quienes han trabajado en la intervención de pacientes con cáncer de cabeza y cuello. Se utilizaron como motores de búsqueda Pubmed, Medline y Biblioteca de la Universidad Santa Paula; los descriptores utilizados fueron head and neck cancer, OR cancer treatment OR chemotherapy OR radiotherapy AND dysphagia OR disturbances in voice OR phonation OR deglution OR breathing OR vocal cords AND therapy. Se revisaron un total de 25 artículos y se incluyeron 11 que cumplieron los criterios de inclusión. Resultados: según los artículos revisados y el criterio de las terapeutas del lenguaje de Costa Rica, en la intervención de las alteraciones deglutorias, se utilizan técnicas compensatorias (cambios posturales, la modificación de consistencia, la modificación del volumen y velocidad de presentación del bolo) y técnicas de rehabilitación (maniobras deglutorias, ejercicios específicos para la movilidad orofacial, técnicas de facilitación y terapia miofuncional), cuanto a las alteraciones de voz, se utilizan técnicas como la higiene vocal, trabajo vocal y las maniobras posturales. Conclusión: en Costa Rica las cifras del cáncer de cabeza y cuello equivalen al 3,5\% en la población masculina y un $12 \%$ en las mujeres. Estos pacientes generalmente reciben tratamiento médico, el cual ocasiona efectos secundarios que pueden provocar alteraciones deglutorias y de la voz. El terapeuta del lenguaje cumple una función clave para pacientes sobrevivientes de cáncer de cabeza y cuello, ya que debe identificar y rehabilitar las alteraciones deglutorias y de la voz resultantes de la patología o su tratamiento, mediante técnicas compensatorias y de rehabilitación, técnicas de higiene vocal, trabajo vocal y maniobras posturales.

Palabras clave: cáncer de cabeza cuello, terapia de lenguaje, deglución, disfagia, fonación. 


\section{Abstract}

Introduction: in Costa Rica, the incidence rate of head and neck cancer for 2016 was of 68,3\%, with a mortality rate of $25,4 \%$. This type of cancer originates in the airways, thyroid and, salivary glands, and generates significant disturbances in swallowing and the production of the voice. The following work seeks to define the speech therapist's intervention in swallowing and speech disturbances in patients with head and neck cancer. Methodology: a bibliographical review was carried out based on review articles written in English and Spanish and books, published from 2000 to 2019. Experts point of view was recollected, for example, speech therapists who had worked in the intervention of patients with head and neck cancer. The bases used were PubMed, Medline, Santa Paula University's Library; the descriptors used were head and neck cancer, OR cancer treatment OR chemotherapy OR radiotherapy AND dysphagia OR disturbances in voice OR phonation OR deglution OR breathing OR vocal cords AND therapy. A total of 25 articles were revised, and 11 were included that complied with the terms of inclusion. Results: according to the opinions of the speech therapists in Costa Rica, the techniques used in the intervention of swallowing disturbances are compensatory techniques (postural maneuvers, modification of consistency, modification of volume and speed of presentation of the bolus) and rehabilitative techniques (swallowing maneuvers, exercises specifically for the orofacial mobility, facilitation techniques, and myofunctional therapy). For speech disturbances, techniques like vocal hygiene, vocal work and the postural maneuvers are used. Conclusion: in Costa Rica, the quantity of head and neck cancer cases is equivalent to $3,5 \%$ of the male population and $12 \%$ of the female population. These patients generally receive medical treatment, which can have secondary effects that cause disturbances in communication, breathing, and swallowing. The speech therapist fulfills a key function for survivors of head and neck cancer, as it identifies and rehabilitates the disturbances in communication, breathing, and swallowing caused by the pathology or its treatment, with the use of compensatory and rehabilitative techniques, methods for vocal hygiene, vocal work, and postural maneuvers.

Keywords: head and neck cancer, speech therapy, swallowing, dysphagia, phonation.

\section{Introducción}

El cáncer se define como un grupo de enfermedades caracterizadas por el crecimiento excesivo y/o descontrolado de células, las cuales llegan a invadir y a dañar tejidos u órganos hasta finalmente provocar la muerte del individuo. La teoría genética propone que estas alteraciones se deben a mutaciones somáticas y la teoría epigenética sugiere que son a causa de alteraciones metabólicas ${ }^{1}$.

Según el Sociedad Americana de Oncología Clínica $^{2}$ existen tres tipos de células principales donde se desarrolla el cáncer, por lo tanto, este indica que el cáncer de cabeza y cuello (CCC) se origina en las células epiteliales escamosas, encargadas de revestir las superficies húmedas y mucosas del interior de la cavidad oral hasta el esófago, exceptuando las áreas del sistema nervioso central.

A nivel mundial el CCC representa aproximadamente el $3 \%$ de tumores malignos. En Costa Rica las cifras del CCC equivalen al 3,5\% todos los tipos de cáncer en la población masculina y un $12 \%$ en las mujeres; siendo la glándula tiroides el más frecuente en ambos sexos ${ }^{3}$, con una tasa de 17,0 por 100000 y 91,27 x 100000 respectivamente en cada sexo. Las mujeres entre las edades de 50 a 54 años son las más propensas a presentar 
cáncer de tiroides, siendo el cuarto tipo de cáncer más frecuente en esta población; en el caso de los hombres es el noveno más común entre los diferentes tipos de cáncer. La provincia donde hay mayor población con CCC es San José, donde no hay un registro exacto de los motivos y comparaciones entre zonas demográficas, sin embargo, analizando los factores de riesgo que produce un cáncer, la capital es de las zonas con mayor contaminación ambiental y donde las personas llevan un estilo de vida muy diferente al de las zonas rurales, principalmente en la alimentación ${ }^{3}$.

El CCC según la zona en la que inicia, se clasifica de la siguiente manera:

1. Cáncer de la vía aero-digestiva superior: tumores que afectan la fisonomía del paciente, así como la calidad de vida mediante la presencia de alteraciones en la comunicación, respiración, y alimentación ${ }^{4}$. El cáncer de la vía aero-digestiva se clasifica en:

- Cáncer de senos paranasales y cavidad nasal: Son poco frecuentes, se localizan en el hueso maxilar, seguido por las fosas nasales, hueso etmoides y finalmente hueso esfenoides y hueso frontal ${ }^{4}$.

- Cáncer de cavidad oral: Se presenta en los labios, el revestimiento interior de los labios y la mucosa bucal, los dientes, las encías, las dos terceras partes anteriores de la lengua, la base de la boca debajo de la lengua y en el paladar duro ${ }^{5}$.

- Cáncerdelaringe:tumoraciónmalignaque afecta las cuerdas vocales y estructuras adyacentes. Se denominará según la sección de la laringe que se encuentre afectada en cáncer supraglótico, cáncer glótico y cáncer subglótico. Siendo el de mayor frecuencia el cáncer glótico ${ }^{6}$.

- Cáncer de faringe: Se puede presentar en alguna de sus tres regiones: nasofaringe, orofaringe e hipofaringe ${ }^{6}$.

2. Cáncer de tiroides: es el tumor más común de los malignos originados en órganos endocrinos y comprende un grupo de tumores que son diferentes clínicamente, epidemiológicamente y en cuanto a pronóstico $^{7}$. Es un tumor relativamente infrecuente, usualmente responde muy bien al tratamiento y frecuentemente se puede curar con cirugía y yodo radiactivo. Incluye: cáncer papilar de tiroides, cáncer folicular de tiroides, cáncer medular de tiroides y cáncer anaplásico de tiroides.

3. Cáncer de glándulas salivales: son poco frecuentes, pueden comenzar en cualquiera de las glándulas salivales de la boca, del cuello o de la faringe. Entre ellas se encuentra la parótida, la sublingual y la submandibular, así como las glándulas salivales pequeñas (menores). Sin embargo, el $75 \%$ de los tumores en glándulas salivales mayores ocurren en la parótida ${ }^{8}$.

Según Alvarenga et $\mathrm{al}^{9}$, el cáncer oral es uno de los principales CCC con un $40 \%$, el cáncer faríngeo representa un $15 \%$, el cáncer laríngeo un $25 \%$ y los tumores restantes se presentan en otros sitios (glándulas salivales y tiroides). Por lo que la mayoría presentan alteraciones del habla, voz y/o deglución. Los tumores de lengua, amígdalas o faringe están más relacionados con la deglución y los tumores de laringe o tiroides con problemas de voz.

A pesar de que aún no es posible determinar los factores de riesgo generales con exactitud, el doctor 
Felipe Cardemil ${ }^{10}$, define que un $75 \%$ de los casos se deben al tabaco y el alcohol, aunque existen otros factores como la edad y la infección del Virus del Papiloma Humano (VPH) y el Virus de EpsteinBarr (VEB).

El tratamiento del CCC lo va a determinar el sitio y la histopatología del tipo de cáncer. Puede abarcar desde la radioterapia o cirugía, los pacientes en los estadios tempranos y en los casos más avanzados incluyen la combinación de radioterapia, con cirugía y quimioterapia.

El equipo interdisciplinario aparte de los médicos va estar conformado principalmente por:

- Odontólogos: ya que las piezas dentales, se afectan con los tratamientos médicos.

- Nutrición: Su función va ser mantener el peso y un balance nutricional en todo el proceso.

- Psicólogos: atención a la salud mental y sobrellevar el duelo.

- Terapeuta del lenguaje: la intervención de Terapia del Lenguaje en pacientes con CCC consiste en identificar y rehabilitar las complicaciones deglutorias y de la voz, a fin de lograr la eficacia de la comunicación, la reeducación motora, manejo del alimento, y la protección de la vía aérea ${ }^{11}$.

A nivel nacional, no existe un protocolo de intervención en cuanto a patologías de deglución y voz en pacientes con CCC; solamente existe un manual por parte de la Caja Costarricense del Seguro Social, que determina las "Normas del Cáncer en Costa Rica" ${ }^{12}$. Pese a la falta de una intervención protocolizada, hay diferentes test que permiten la evaluación del sistema estomatognático, voz, habla, deglución y comunicación, ampliamente utilizados por los terapeutas del lenguaje. Dentro de las evaluaciones estandarizadas se encuentran el PEMO, el SAFE y el test de Boston; y las no estandarizadas como las fichas de voz y barridos que incluyen la alimentación y respiración. Todas ellas serán abordadas en los resultados.

En cuanto a servicios de salud públicos en Costa Rica, solamente el CENARE, Hospital México, Hospital Calderón Guardia y el Hospital Blanco Cervantes cuentan con terapeutas del lenguaje, de los cuales un $25 \%$ han intervenido en pacientes con $\mathrm{CCC}$, indicando que los pacientes son referidos entre 2 a 4 meses después de la intervención médica.

Por lo anterior, el objetivo de este artículo es definir la intervención del terapeuta del lenguaje en las alteraciones deglutorias y de la voz en pacientes con CCC, donde no existe un protocolo como tal para el terapeuta del lenguaje en el manejo de este tipo de patologías.

\section{Metodología}

Se realizó un estudio de revisión narrativa basada en la evidencia existente sobre la detección e intervención de alteraciones deglutorias y de la voz en pacientes con CCC, por parte del terapeuta del lenguaje.

El estudio se basó en artículos científicos y libros publicados del año 2000 en adelante y bases de datos del 2015 en adelante de la Caja Costarricense de Seguro Social, del Instituto Nacional de Estadísticas y Censos y del Ministerio de Salud, así como estudios a nivel nacional e internacional; obtenidos de motores de búsqueda específicos como: PubMed, Medline, Biblioteca de la Universidad Santa Paula y bases de datos de acceso libre con información de tesis. Todos estos estudios de idiomas inglés y el español. Así mismo, se realizó un cuestionario acudiendo al criterio de expertos, este fue aplicado a terapeutas del 
lenguaje quienes han trabajado en la intervención de pacientes con CCC tanto a nivel público como privado, con experiencia de entre 4 a 17 años.

La búsqueda arrojó un total de 25 artículos que abordaban intervenciones terapéuticas de la voz y la deglución en pacientes con CCC. Además, se acudió al criterio de expertos en terapia del lenguaje que trabajan a nivel nacional tanto en servicios públicos como privados, y que atienden pacientes con CCC.

\section{Discusión de Resultados}

\section{Alteraciones deglutorias en pacientes con cáncer de cabeza y cuello}

Antes de conocer cuáles son las alteraciones que puede presentar una persona con CCC, es importante definir el proceso de deglución. Con el fin de identificar cada una de las alteraciones según las estructuras implicadas.

Logemann 13 define la deglución como una actividad neuromuscular compleja y rápida (de 3 a 8 segundos) de la cavidad bucal, faringe, y laringe, cuyo objetivo es aplicar presión a los alimentos, propulsando estos desde la boca hacia el esófago. En ella participan 30 músculos y 6 pares de nervios encefálicos: trigémino- $\mathrm{V}$, facial-VII, glosofaríngeoIX, vago-X, accesorio espinal-XI e hipogloso-XII.

Para que se efectúe la deglución propiamente dicha, debe de pasar por 4 etapas las cuales realizan funciones voluntarias e involuntarias ${ }^{13,14,15}$. Estas se clasifican en:

- Etapa 0. Preparación extraoral o anticipatoria: para algunos autores, no entra como parte de la deglución. Sin embargo, es de gran importancia porque por medio de ella pueden recuperar determinados automatismos ante estímulos sensoriales, anticipa al paciente y se desencadena la estimulación salival. Por lo tanto, en un paciente paciente que ha recibido radioterapia es difícil que pueda iniciar esta etapa de manera correcta.

- Etapa I. Oral: es una etapa voluntaria y consciente, se subdivide en oral preparatoria y oral propiamente dicha. Durante la etapa oral preparatoria se inicia con la prensión de los alimentos, durante esta etapa el alimento se mezcla con la saliva y se transforma en un bolo homogéneo. Su duración depende del tiempo de la masticación. En la etapa oral propiamente dicha se inicia con el posicionamiento del bolo alimenticio en la lengua y finaliza con la eyección del alimento hacia la faringe. Esta etapa dura menos de un minuto.

- Etapa II. Faríngea: es consciente pero involuntaria, también conocida como automática-refleja. El bolo alimenticio desencadena una serie de reflejos: cierre del velo del paladar, anteriorización de la pared posterior de la faringe, compresión del bolo contra el dorso de la lengua, cierre de glotis protegiendo vía aérea y la respiración se suspende temporalmente. Esta etapa dura en promedio un segundo.

- Etapa III. Esofágica: es inconsciente e involuntaria. Se presenta por medio de las contracciones peristálticas, que tienen inició con las contracciones de la faringe, presión del tono esfinteriano y atracción inducida por la elevación de la laringe conduciendo el bolo desde el esófago hacia el estómago.

Por lo tanto, si se presenta alguna alteración desde el momento en que se le presentan los 
alimentos al paciente hasta que pasen al estómago se denominará disfagia. Esta se puede clasificar según su etiología y su localización ${ }^{14}$.

- Según su etiología

1. Neuromuscular: se origina en el sistema nervioso central.

2. Mecánica: conocida como disfagia funcional.

- Según su localización

1. Disfagia oral: implica el posicionamiento del alimento a la cavidad bucal y la masticación.

2. Disfagia orofaríngea: dificultad en el momento que se da el transporte del bolo alimenticio a través de la faringe hacia el esófago.

3. Disfagia esofágica: dificultad de transportar el bolo al estómago debido a una alteración en las contracciones peristálticas.

Las alteraciones deglutorias relacionadas al CCC más frecuentes son las aspiraciones, los movimientos compensatorios de cabeza y la presencia de residuos de alimentos después de la deglución, las cuales se definen en la Figura 1. Según los expertos en terapia del lenguaje, estas alteraciones se rehabilitan en un lapso de 3 a 6 meses aproximadamente.

Según un estudio realizado en España por Martín Villares et al ${ }^{17}$, se determinó que los pacientes con tumores de laringe e hipofaringe presentaron mayor alteración en la deglución antes de recibir algún tratamiento como la cirugía, radioterapia o quimioterapia, y los pacientes con tumores en la cavidad oral y orofaríngea tuvieron mayor dificultad después de recibir los tratamientos. Este estudio indica que, tanto pre-tratamiento como posttratamiento, requieren de intervención terapéutica para manejar y establecer una alimentación segura.

\section{Alteraciones de voz presentes en pacientes con cáncer de cabeza y cuello}

Antes de conocer las alteraciones presentes en la voz se debe de conocer la fisiología y los órganos que intervienen en esta, como explica Le Huche et $\mathrm{al}^{18}$, los órganos utilizados para el habla poseen funciones preexistentes como lo son la respiración y la masticación; sin embargo, se aprovechan estas estructuras para la producción del habla y la voz, volviendo estas funciones secundarias. Para su estudio, se divide el aparato vocal en tres partes: los fuelles (permite el movimiento del aire), el vibrador (órgano principal de la voz) y los resonadores (pabellón faríngobucal y cavidades anexas).

Según Calais Germain et $\mathrm{al}^{19}$, los sonidos del habla son similares a las notas musicales en que se ven caracterizados por diversos componentes como lo son: duración, altura, intensidad y timbre.

Jackson Menaldi ${ }^{20}$ define que las razones por las que ocurren afectaciones de la voz son debido a un daño o limitación en las estructuras que permiten su producción. Estas afectan tanto la voz, la deglución y/o la respiración de forma variable, y no son acompañadas por síntomas neurológicos que puedan indicar una alteración central o bulbar.

Las alteraciones presentes en la voz se denominan disfonías. Le Huche et $a^{21}$ las define como un trastorno momentáneo o duradero de la función vocal considerado como tal por la propia persona o por su entorno. Por lo común, pero no obligatoriamente, se traduce por alteración de uno o varios parámetros de la voz, que son, por orden de frecuencia: el timbre, la intensidad, y la altura tonal.

Susanibar et al ${ }^{15}$ mencionan que las disfonías se clasifican según su etiología en: 


\section{Penetraciones}

- Consiste en la entrada de alimento en la vía aérea (VA), sin embargo, no sobrepasa las CV.

\section{Aspiraciones}

- Consiste en la entrada de alimento directamente en la VA, sobrepasando las CV. En algunos pacientes el reflejo esta inhibido o no se presenta con fuerza para expulsar el alimento.

- 1. Antes de la deglución (AD): existe un mal control del bolo, en donde parte del alimento en el momento del desmenuzamiento oasa hacia la faringe.

-2. Durante la deglución (DD): el reflejo está presente, sin embargo, la epiglotis no realiza el cierre completo o es retrasado, esta deficiencia se asocia a un cierre incompleto de las cuerdas vocales.

- 3. Después de la deglución (DED): el peristaltismo laríngeo es débil lo cual disminuye y arrastra con dificultad el bolo, el reflejo de deglución es correcto, queda restos de alimetos en las paredes faríngeas, produciendo un éxtasis vallecular; provocando la aspiración.

\section{Movimientos compensatorios de cabeza}

- Movimientos compensatorios durante la deglución, caracterizados por el estiramiento de los músculos del cuello, proyectándose la cabeza hacia atrás. Una posibles causa es la masticación ineficiente.

Presencia de residuos de alimentos después de la deglución

- Puede ocurrir debido a la presencia de buccinadores en hiperfunción, ala disminución en la cantidad de saliva y la alteración en la movilidad y7o la percepción de la lengua. Se considera que los alimentos muy secos y harinosos favorecen la presencia de residuos después de la deglución.

\section{Figura 1. Alteraciones deglutorias más frecuentes en pacientes con cáncer de cabeza y cuello}

Fuente: elaboración propia basada en las referencias ${ }^{15,16}$

- Disfonías orgánicas: se muestra independiente al uso de la voz, es causada por lesiones en los órganos de la fonación debidas a causas congénitas, inflamatorias y traumáticas.

- Disfonías funcionales: relacionada a cómo se utiliza la voz, se divide en disfuncionales y funcionales estructurales.

- Disfonía disfuncional: se deben a la sobrecarga de la actividad vocal, en ausencia de cambios orgánicos.

- Disfonía funcional estructural: ocurre como resultado de variaciones anatómicas no ideales para la fonación normal, tales como los cambios estructurales de la laringe, del sistema respiratorio, de las cavidades anexas y trastornos discretos del movimiento.

- Disfonías orgánico-funcionales: alteraciones orgánicas como consecuencia de un uso inadecuado de la voz.

En cuanto a las alteraciones de voz relacionadas al cáncer de cabeza y cuello más frecuentes son las alteraciones de estructuras laríngeas, disturbios respiratorios, trastornos posturales, trastornos relacionados con la intensidad del habla, trastornos de la calidad de voz y las parálisis periféricas de cuerdas vocales. Las cuales se definen en la Figura 2. Según expertos estas alteraciones se rehabilitan en un lapso de 3 a 6 meses. 


\section{Alteraciones en las estructuras laríngeas}

-Pequeños cambios en la configuración de la laringe, variaciones anatómicas y/o malformaciones menores

\section{Disturbios respiratorios}

- Mala coordinación entre la respiración y la fonación

- La persona no administra bien el aire al hablar o cantar.

Trastornos posturales

- Todo cambio postural adquirido por la persona afecta la eficacia respiratoria, articulatoria y vocal.

\section{Trastornos relacionados con la intensidad del habla}

- Comunicaciones con fuerte intensidad en ambiente ruidoso sin el uso de una técnica vocal, sin calentamiento previo o sin utilización de equipos de amplificación de sonidos.

\section{Trastornos de la calidad de voz}

-Relacionados con la percepción auditiva de ronquera, aspereza, soplo, astenia, tensión e inestabilidad vocal.

\section{Parálisis periféricas de cuerdas vocales (unilateral y bilateral)}

- Unilateral: sólo uno de los repliegues vocales se encuentra paralizada.

- Bilateral: ambos repliegues vocales se ven afectadas.

- Las CV pueden verse paralizadas en distintas posiciones:

-1. Fijación en línea mediana: esta puede ser la más peligrosa si es una parálisis

bilateral, ya que no permite el paso del aire.

-2. Fijación en línea lateral: la cuerda está en su posición más abierta.

-3. Fijación en línea intermedia: la cuerda se mantiene en una posición entre la mediana y la lateral.

Figura 2. Alteraciones de la voz en pacientes con cáncer de cabeza y cuello

Fuente: elaboración propia basada en las referencias ${ }^{15,23,25}$

\section{Intervención de la terapia del lenguaje en las alteraciones deglutorias en pacientes con cáncer de cabeza y cuello}

Según Peña Casanova ${ }^{22}$, la terapia del lenguaje es una disciplina que se define como aquella que engloba el estudio, la prevención, evaluación, diagnóstico, y tratamiento de las alteraciones en la comunicación humana. Así como la encargada en la intervención de áreas como: habla, voz, lenguaje oral y escrito, cognición, orales no verbales y audición.

El terapeuta del lenguaje se encuentra en la obligación de evaluar al paciente antes de iniciar cualquier proceso de intervención, con el objetivo de considerar la técnica de intervención adecuada según los síntomas que presente en cuanto a las alteraciones deglutorias y de voz, ya que, al ser tan complejo no se puede definir una técnica específica en general, debido a que los síntomas varían según la persona y el estadiaje oncológico en la que se encuentre ${ }^{3}$.

El objetivo principal de la intervención de terapia del lenguaje en las alteraciones deglutorias es rehabilitar la alimentación oral, logrando 
una deglución segura, mediante técnicas de rehabilitación y/o compensatorias; permitiendo la eliminación de aspiraciones y disminución de los residuos orales o faríngeos tras la aspiración ${ }^{24}$.

Existen diversas técnicas de compensación y rehabilitación para la intervención de Terapia del Lenguaje en las alteraciones deglutorias. No obstante, las técnicas más utilizadas son las técnicas compensatorias (cambios posturales, modificación del volumen y de velocidad de presentación del bolo y modificación de consistencia) y técnicas de rehabilitación (maniobras deglutorias, los ejercicios de movilidad orofacial y la terapia miofuncional). A continuación, se explican cada una de estas técnicas.
- Técnicas de compensación

El objetivo es eliminar los síntomas del paciente, pero no cambia necesariamente la fisiología de su deglución.

1. Cambios posturales: compensan las deficiencias deglutorias evitando el riesgo de aspiración y facilitando el paso del alimento hacia el esófago ${ }^{26}$. Son de forma temporal hasta que la deglución se recupere o las técnicas del tratamiento mejoran la función motora orofaríngea. En la Figura 3 se definen las posturas utilizadas en pacientes con disfagia.

2. Modificación del volumen y velocidad de presentación del bolo: el volumen del

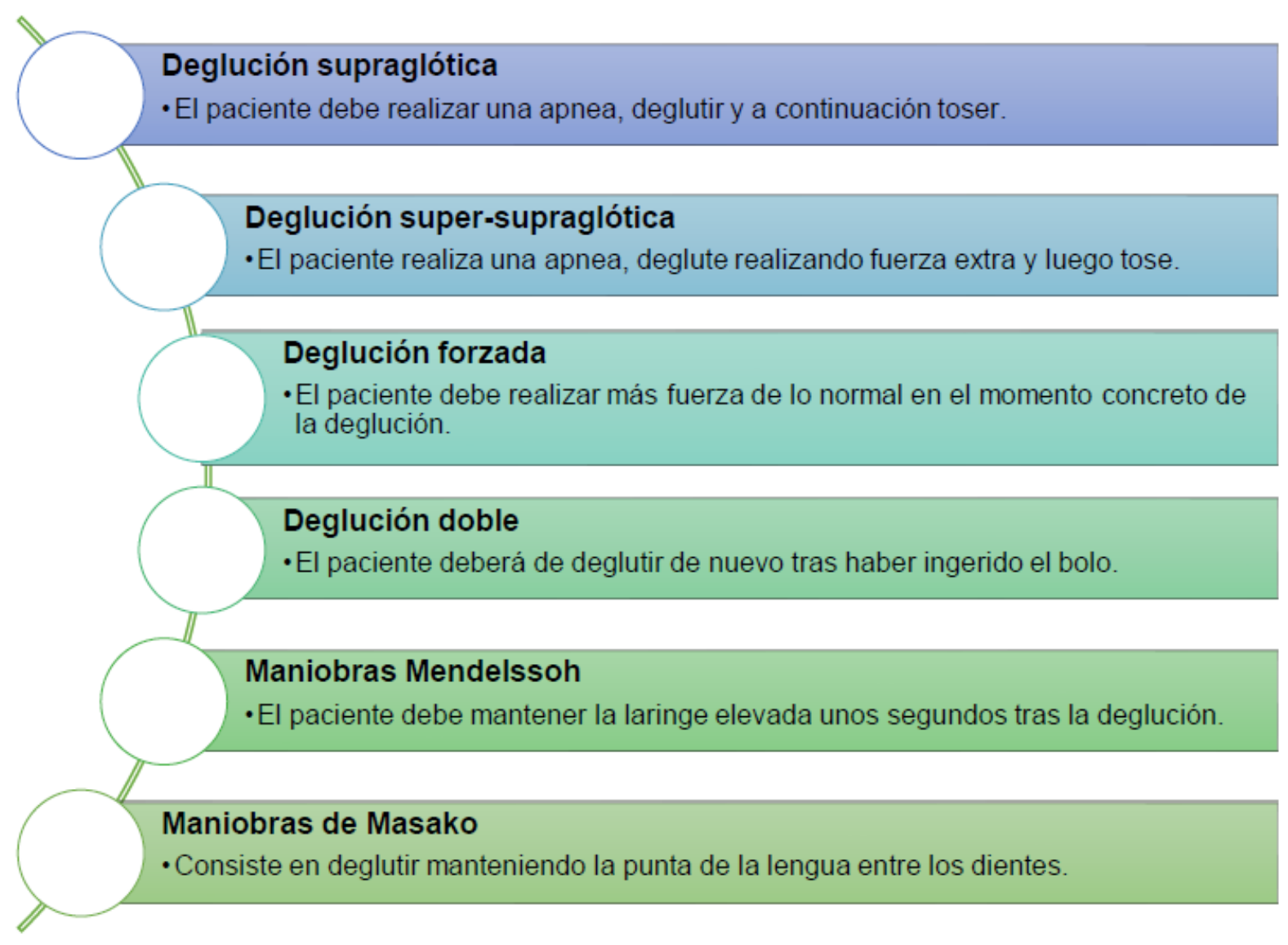

Figura 3. Posturas utilizadas en pacientes con disfagia

Fuente: elaboración propia basada en la referencia ${ }^{26}$ 
alimento que el paciente pueda deglutir dependerá de la facilidad y seguridad de este. En individuos con debilidad en la deglución faríngea que precisan dos o tres degluciones por bolo, la compensación será tan simple como dar bolos más pequeños a una velocidad menor para disminuir el riesgo de aspiración por acúmulo de comida en la faringe ${ }^{26}$.

3. Modificación de consistencia: esta técnica consiste en que el alimento sea homogéneo, evitando grumos y espinas, que sea jugoso y de fácil masticación. Se deben evitar dobles texturas con mezclas de líquido y sólido. Incluir la máxima variación de alimentos para evitar la rutina y procurar que las condiciones organolépticas sean atractivas ${ }^{27}$.

- Técnicas de rehabilitación

El objetivo es modificar la fisiología y mejorar el funcionamiento de la deglución, intentando recuperar determinados automatismos para la independencia de la persona. Las siguientes técnicas son basadas en Del Burgo ${ }^{14}$, Bascuñana ${ }^{26}$, Morales Martínez ${ }^{28} \mathrm{y}$ Bleeckx ${ }^{29}$.
1. Maniobras deglutorias: se aplican para aumentar la seguridad y la eficacia del proceso deglutorio. Permitiendo controlar de forma voluntaria ciertos aspectos de la deglución, reduciendo o eliminando las alteraciones presentes en la disfagia. Estas maniobras se definen en la Figura 4.

2. Ejercicios específicos: ejercicios destinados a la estabilidad y movilidad orofacial. Existen dos tipos: los masajes para la hipotonía y los ejercicios para la movilidad orofacial. Sin embargo, en la Figura 5 se determinan solamente los ejercicios más utilizados en las alteraciones deglutorias en pacientes con cáncer de cabeza y cuello.

3. Técnicas de facilitación: ejercicios destinados a potenciar la funcionalidad de la musculatura afectada. Basados en ejercicios de estimulación, estiramientos, manipulación y resistencia.

4. Terapia Miofuncional: su objetivo es obtener el tono idóneo en los músculos afectados, con posterioridad a recuperar la actividad motora, así como la movilidad y fuerza de las estructuras implicadas en la deglución. Antes

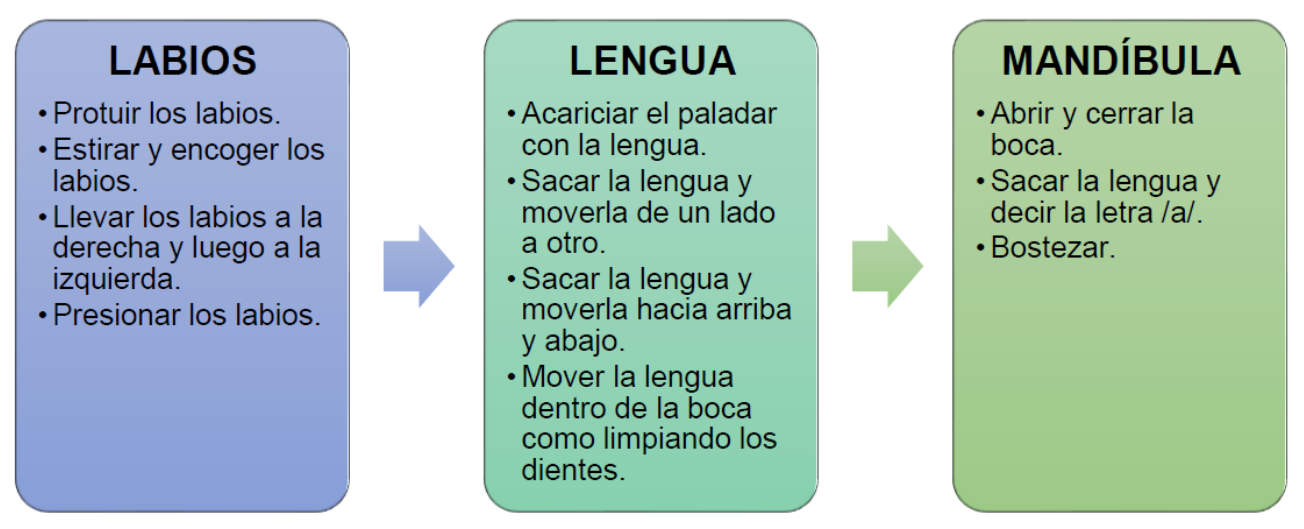

Figura 4. Maniobras deglutorias

Fuente: elaboración propia basada en las referencias ${ }^{14,26,28,29}$ 
de iniciar con este tipo de terapia, el médico oncológico determina si la zona afectada puede ser tratada con esta estrategia ${ }^{30}$.

\section{Intervención de la Terapia del Lenguaje en las alteraciones de voz en pacientes con cáncer de cabeza y cuello}

Existen diversas técnicas de rehabilitación en las alteraciones de la voz en las cuales es necesario adaptar el programa al individuo, mediante las técnicas como la higiene vocal, técnica de trabajo vocal, técnicas para la voz cantada, técnica del tracto vocal semi-ocluído, técnicas para laringectomizados y técnica en parálisis unilaterales de cuerda vocal. No obstante, las técnicas de mayor uso en las alteraciones de la voz en pacientes con CCC son la higiene vocal, las técnicas de trabajo vocal, y las maniobras posturales, la cual es parte de las
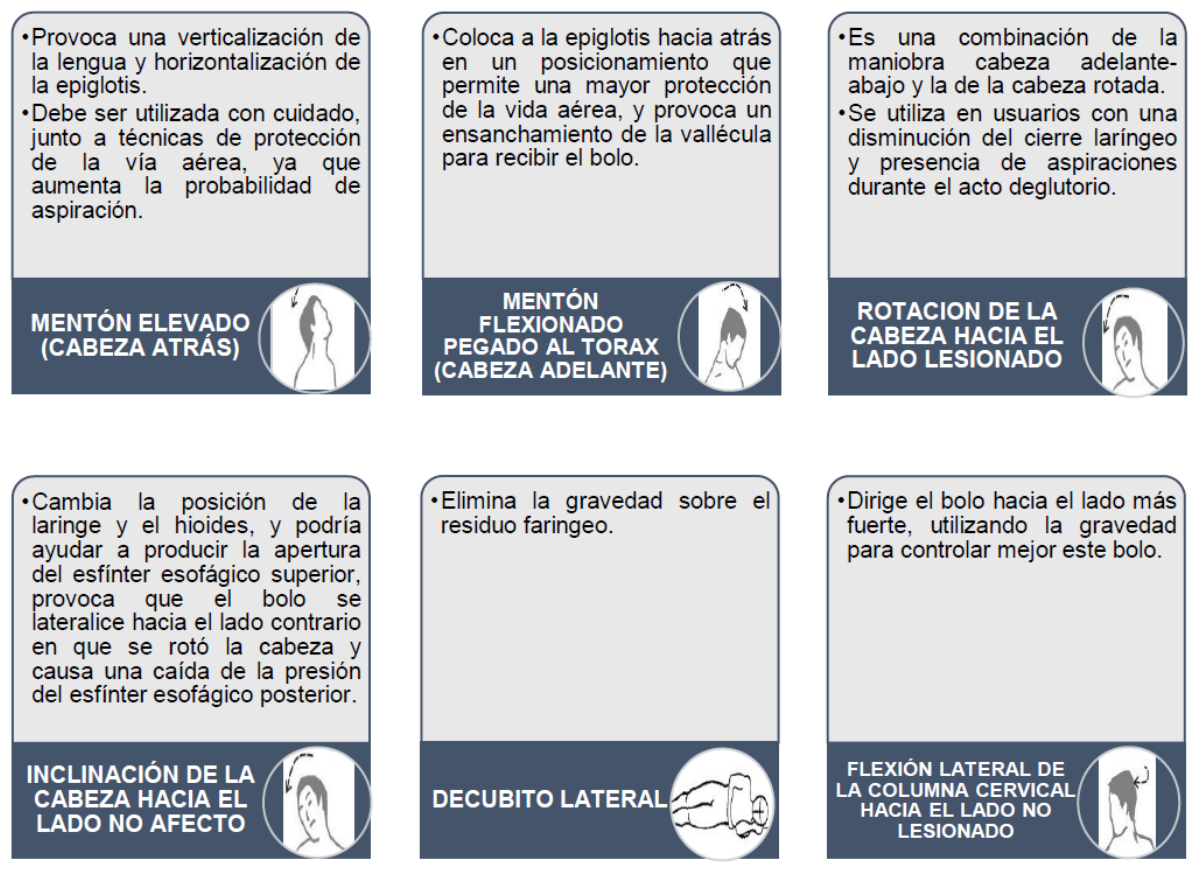

Figura 5. Ejercicios para la movilidad orofacial

Fuente: elaboración propia basada en las referencias ${ }^{29}$

técnicas para laringectomizados. Estas técnicas se definirán a continuación:

\section{- Higiene vocal}

La adquisición de conductas o hábitos positivos demuestran ser beneficiosos para la salud vocal; es importante explicar, conocer, convencer, y potenciar estas conductas saludables, y evitar en lo posible las que perjudiquen la voz. No necesariamente prohibir las conductas perjudiciales, sino buscar un equilibrio de estas con los hábitos positivos.
- Técnicas de trabajo vocal

Estas técnicas son indicadas por Clarke et $\mathrm{al}^{31} \mathrm{en}$ su guía de trabajo multidisciplinario del Reino Unido, incluye aquellas que se basan en la proyección de la voz, el tono, reducción de fatiga y coordinación de la respiración. Su clasificación se observa en la Figura 6.

- Maniobras posturales

Consiste en variaciones de la posición de la cabeza las cuales pueden ayudar a compensar 


\section{Terapia de voz resonante}

- Utilizan sonidos nasales (/m/, /n/, /ñ/) para provocar sensación de vibración de pómulos, fosas nasales, y cabeza; semejante al método masticatorio.

- Útil para el calentamiento vocal, despertando vibraciones interiores y reduciendo tensiones en las disfonias; también usa sonidos y resonancias nasales como /iam/, /dum/.

\section{Ejercicios de fonación vocal}

•Emisión de sonidos vibrantes (/brr/, /rrr/, /trrrl, /blll/).

\section{Técnicas de voz de fritura}

- Utilizada para reducir la tensión vocal en personas que gritan y fuerzan la voz mediante el uso de una voz de tono muy grave que imita el sonido del aceite hirviendo.

\section{Método del acento}

- Técnica que enseña coordinación de la respiración y fonación al paciente de forma lúdica.

- Evita el sobreesfuerzo de los músculos.

- Se basa en un ritmo largo y animado, acompañado de movimiento corporal.

- Se entrena el rango dinámico del acento, primero con onomatopeyas, seguido de palabras, y finalmente con frases, realizando variaciones en la entonación en forma de olas.

\section{Tratamiento vocal de Lee Silverman}

- Utilizada en alteraciones disártricas como la enfermedad de Parkinson, se basa en pedirle al paciente que piense en hablar fuerte.

Masaje y/o manipulación de la laringe
-Aplicada para relajar o para tonificar la musculatura externa en parálisis laríngeas, dependiendo
del caso.

\section{Técnicas de facilitación vocal}

- Se aplican instrumentos como el pito, el tubo de Lax Vox , o el papel vibrante.

- Mejora la vibración, la coordinación fonorespiratoria, aproxima las cuerdas vocales, usado para reeducar la parálisis recurrencial unilateral

\section{Terapia cognitivo-conductuales \\ - Trabaja la respuesta al estrés o problemas psicológicos.

Entrenamiento corporal
- Combate el sobreesfuerzo vocal al mejorar la estabilidad de la cabeza, la columna, y la cintura
escapular.

\begin{tabular}{|l|}
\hline Entrenamiento con biofeedback \\
- Utiliza un espejo o programas de software acústico para escuchar y corregir los errores mientras \\
se visualiza el ataque vocal, los armónicos, o el ruido añadido a la voz. \\
Relajación general y específica \\
• Aplicada en los músculos de la fonación para combatir el sobreesfuerzo vocal. \\
Entrenamiento auditivo \\
- Usado para identificar y eliminar vocalizaciones no deseadas, o técnicas de enmascaramiento. \\
• Útil en disfonías de conversión y de tipo psicológico. Elimina los malos hábitos vocales: como el \\
abuso vocal, el golpe de glotis, el carraspeo, entre otros. \\
Técnica gestual \\
• Aplicada a la modulación de las vocales (cada vocal, un gesto con las manos), al apoyo del sonido \\
(manos que sostienen una bandeja), al uso de resonadores (apoyar los dedos en los pómulos), \\
entre otros gestos.
\end{tabular}

\section{Figura 6. Clasificación de las técnicas de trabajo vocal}

Fuente: elaboración propia basada en la referencia ${ }^{31}$ 
fallas de coadaptación laríngea y desniveles de estructuras. Girar la cabeza al lado facilita la fonación y alimentación. Al llevar la cabeza hacia atrás contribuye a activar el cierre durante la fonación, mientras tanto el paciente deberá de emitir vocales o sílabas. Esta técnica debe ser utilizada en el periodo inicial de la terapia, no debe de ocupar el tiempo de toda la sesión, caracterizándose como facilitador de la emisión ${ }^{16}$.

\section{Conclusiones}

La revisión de la literatura y el criterio de los expertos consultados, nos permite concluir que en Costa Rica los pacientes con CCC generalmente reciben algún tratamiento médico, ya sea radioterapia, quimioterapia o cirugía, los cuales ocasionan efectos secundarios como: cambios en la boca, piel, laringe y/o faringe, dificultad para deglutir, dolor en los oídos, afectación en el sentido del gusto, y dificultad para respirar; hasta el punto de provocar alteraciones deglutorias y de la voz. Es aquí donde el Terapeuta del Lenguaje cumple una función crucial en la intervención de estos pacientes, identificando y rehabilitando dichas alteraciones, con el fin de lograr una eficacia en la comunicación, la reeducación motora, manejo del alimento, y la protección de la vía aérea; mediante técnicas compensatorias y de rehabilitación, técnicas de higiene vocal, trabajo vocal y maniobras posturales.

Los expertos indican además, que aunque es importante que exista una intervención protocolizada, no existe un tratamiento específico que se pueda estandarizar para toda la población y que las alteraciones presentan un lapso de recuperación de entre 3 a 6 meses aproximadamente, lo cual se ve influido por las características que se presenta en cada caso.

\section{Referencias Bibliográficas}

1. Boticario BC, Cascales AM. Innovaciones en cáncer. Madrid: Universidad Nacional de Educación a Distancia; 2012.

2. American Society of Clinical Oncology [Internet]. Virginia: American Society of Clinical Oncology; 2019 [citado el 26 de mayo de 2020]. Head and Neck Cancer: Introduction; [aprox. 3 pantallas]. Disponible en: https://www.cancer.net/cancer-types/ head-and-neck-cancer/introduction

3. Ministerio de Salud (CR), Dirección de Vigilancia de la Salud, Unidad de seguimiento de indicadores de salud. Boletín estadístico de mortalidad por tumores malignos más frecuentes en Costa Rica del año 2014 [Internet]. San José, CR: Ministerio de Salud; 2015 [citado el 26 de mayo de 2020]. Disponible en: https://www.ministeriodesalud. go.cr/index.php/vigilancia-de-la-salud/estadisticas-y-bases-de-datos/estadisticas/estadistica-de-cancer-registro-nacional-tumores/ boletines-de-mortalidad-de-cancer/2789-boletin-de-mortalidad-por-cancer-2014/file

4. Quitral R, Rahal M, Morales I, Daszenies Sickinger C, Vallejos MP. Tumores malignos de nariz y cavidades paranasales: revisión de 11 años. Rev Otorrinolaringol Cir Cabeza Cuello. 2003;63(1):21-28.

5. Werning J. Oral Cancer: Diagnosis, Management, and Rehabilitation. New York: Thieme; 2011.

6. Rodés J, Piqué J, Trilla A, directores. Libro de la salud del Hospital Clínic de Barcelona y de la Fundación BBVA [Internet]. Bilbao: Fundación BBVA; 2007. Disponible en: https://www.fbbva.es/wp-content/ uploads/2017/05/dat/DE_2007_salud_clinic_barcelona.pdf 
7. Gómez Sáez J. Cáncer de tiroides. Barcelona: Elsevier; 2014.

8. Granados García M, Arrieta Rodríguez O, Hinojosa Gómez J. Tratamiento del cáncer. México: Manual Moderno; 2016.

9. Alvarenga L, Torreglosa Ruiz M, Pavarino-Bertelli É, Cabral Ruback M, Maniglia J, Goloni-Bertollo E. Epidemiologic evaluation of head and neck patients in a university hospital of Northwestern São Paulo State. Rev Bras Otorrinolaringol [Internet]. 2008 [citado el 6 de julio de 2019];74(1):68-73. Disponible en: https://www.scielo.br/pdf/rboto/ v74n1/en_a11v74n1.pdf

10. Cardemil F. Epidemiología del carcinoma escamoso de cabeza y cuello. Rev Chil Cir [Internet]. 2014 [citado el 24 de junio de 2020];66(6):614-620. Disponible en: https://scielo.conicyt.cl/pdf/rchcir/v66n6/art17. pdf

11. American Society of Clinical Oncology [Internet]. Virginia: American Society of Clinical Oncology; 2019 [citado el 29 de marzo de 2019]. Oral and Oropharyngeal Cancer: Types of Treatment; [aprox. 8 pantallas]. Disponible en: https://www.cancer.net/cancer-types/oral-and-oropharyngeal-cancer/ types-treatment

12. Piedra Quesada V, comp. Manual de normas para el tratamiento de cáncer en Costa Rica [Internet]. San José, CR: Imprenta Nacional; 2014 [citado el 26 de mayo de 2020]. Disponible en: https://www. imprentanacional.go.cr/editorialdigital/libros/ textos\%20juridicos/manual_contra_el_cancer_edincr.pdf

13. Logemann J. Fisiología y fisiopatología de la deglución. Clínicas Otorrinolaringólogas de Norteamérica; 1988.
14. Del Burgo González de la Aleja G. Rehabilitación de problemas de deglución en pacientes con daño cerebral sobrevenido. 2a ed. Madrid: EOS; 2004.

15. Susanibar F, Marchesan I, Parra D, Dioses A. Tratado de evaluación de motricidad orofacial y áreas afines. Madrid: EOS; 2014.

16. Barros A, Arakawa L, Tonini M, Carvalho V. Fonoaudiologia em cancerología [Internet]. Sao Paulo: Fundacao Oncocentro; 2000. Disponible en: http://bvsms.saude. gov.br/bvs/publicacoes/fonoaudiologia_cancer.pdf

17. Martín Villares C, Tapia Risueño M, San Román Carbajo J, Fernández Pello ME, Domínguez Calvo J. Disfagia pretratamiento en pacientes con cáncer avanzado de cabeza y cuello Nutr Hosp [Internet]. 2003 [citado el 22 de abril de 2019];18(5):238-242. Disponible en: http://scielo.es/pdf/nh/v18n5/ original1.pdf

18. Le Huche F, André Allali A. La voz. Tomo 1. Anatomía y fisiología de los órganos de la voz y del habla. Barcelona: Masson; 1993.

19. Calais Germain B, Germain F. Anatomía para la voz. Barcelona: La Liebre de Marzo; 2013.

20. Jackson Menaldi MCA. La voz patológica. Buenos Aires: Médica Panamericana; 2002.

21. Le Huche F, Allali A. La voz. Tomo 2. Patología vocal: semiología y disfonías disfuncionales. Barcelona: Masson; 1993.

22. Peña Casanova J. Manual de logopedia. 4a ed. Barcelona: Elsevier; 2014. 
23. Chen X, Wan P, Yu Y, Li M, Xu Y, Huang $P$, Huang $Z$. Types and timing of therapy for vocal fold paresis/paralysis after thyroidectomy: a systematic review and meta-analysis. J Voice. 2014;28(6):799-808. doi: 10.1016/j.jvoice.2014.02.003.

24. Casado JC. Clínica Otorrinolaringológica Marbella [Internet]. Málaga: Clínica Otorrinolaringológica Marbella; 2012 [citado el 1 de abril de 2019]. Parálisis de Cuerda Vocal; [aprox. 5 pantallas]. Disponible en: https://www.otorrinomarbella.com/paralisis-de-cuerda-vocal/

25. Arroyo Sanabria MJ, Madriz Hernandez J, Sequeira Valverde C. Relación entre el tratamiento de quimioterapia y/o radioterapia y las complicaciones en cavidad oral en personas con enfermedad oncológica avanzada en cabeza y cuello en Costa Rica [Tesis de Maestría en Cuidados Paliativos]. San José, CR: Universidad Santa Paula; 2018.

26. Bascuñana Ambrós H, Gálvez Koslowski S. Tratamiento de la disfagia orofaríngea. Rehabilitación (Madr) [Internet]. 2003 [citado el 3 de mayo de 2019];37(1):40-54. Disponible en: https://futurofonoaudiologo. files.wordpress.com/2014/10/tratamiento-de-la-disfagia-orofarc3adngea.pdf

27. González Alted C, Casado Romo MP, Gómez Blanco A, Pajares García S, Dávila Acedo RM, Barroso Pérez $L$, et al. Guía de nutrición para personas con disfagia [Internet]. Madrid: IMSERSO; 2017. Disponible en: https://www.imserso.es/InterPresent1/groups/imserso/documents/binario/402017002_guia_nutricion_perso.pdf

28. Morales Martínez F, editor. El arte de envejecer bien: consejos prácticos. San José, CR: EDNASSS; 2018.
29. Bleeckx D, Postiaux G. Disfagia evaluación y reeducación de los trastornos de la deglución. Madrid: McGraw-Hill; 2004.

30. Susanibar F, Castillo J, Douglas C, coordinadores. Motricidad Orofacial fundamentos basados en evidencia. Madrid: EOS; 2016.

31. Clarke P, Radford K, Coffey M, Stewart M. Speech and swallow rehabilitation in head and neck cancer: United Kingdom National Multidisciplinary Guidelines. J Laryngol Otol. 2016 [citado el 3 de mayo de 2019];130(S2):176-180. doi: 10.1017/ S0022215116000608 